\title{
In vitro Evaluation of Proton Motive Force-Dependent Efflux Pumps Among Multidrug Resistant Acinetobacter baumannii Isolated From Patients at Tehran Hospitals
}

\author{
Parisa Nikasa ${ }^{1}$, Ahya Abdi-Ali ${ }^{1,}$, Azadeh Rahmani-Badi ${ }^{1}$, Arif Al-Hamad ${ }^{2}$ \\ ${ }^{1}$ Department of Biology, Faculty of Science, Al-zahra University, Tehran, IR Iran \\ 2 Divisions of Clinical Microbiology and Infection Prevention and Control, Qatif Central Hospital, Qatif, Saudi Arabia \\ ${ }^{*}$ Corresponding author: Ahya Abdi-Ali, Department of Biology, Faculty of Sciences, Alzahra University, Tehran, IR Iran. Tel: +98-2188058912, Fax: +98-2188058912, E-mail: abdialya@ \\ alzahra.ac.ir.
}

Received: Jun 17, 2012; Revised: July 31, 2012; Accepted: August 09, 2012

\begin{abstract}
Background: Multidrug-resistant (MDR) strains of Acinetobacter baumannii have been increasingly reported as a major cause of nosocomial infections, and have created major therapeutic problems worldwide.

Objectives: The aim of the present study was to evaluate the role of proton motive force (PMF)-dependent efflux mechanism in the multiple resistance phenotype of $A$. baumannii clinical strains.

Materials and Methods: A total of 65 A. baumannii clinical strains were collected from hospitals in Tehran. These were tested for antimicrobial susceptibility using disc agar diffusion and broth microdilution methods. Active efflux was assessed by ethidium bromide accumulation assays. Further evaluations were performed by the determination of the minimum inhibitory concentrations and the accumulation of ciprofloxacin against selected MDR A. baumannii in the presence and absence of carbonyl cyanide m-chlorophenylhydrazone (CCCP), an inhibitor of PMF.

Results: Ninety five percent of strains were MDR, with high rate of resistance to ciprofloxacin (92.3\%), gentamicin (89.2\%), and ceftazidime (93.8\%).Colistin and imipenem were the most effective antibiotics with resistance rates of $1.5 \%$ and $44.6 \%$, respectively. MDR strains showed a four-fold reduction in the MIC of ciprofloxacin when tested in the presence of the efflux pump inhibitor. The addition of CCCP led to a significant increase in the accumulation of ethidium bromide and ciprofloxacin.

Conclusions: PMF-dependent efflux mechanism appears to play an important role in the MDR phenotype of A. baumannii clinical strains.
\end{abstract}

Keywords: Acinetobacter baumannii; Multidrug Resistant; Efflux Pump Inhibitor; Drug Accumulation

\section{Background}

Acinetobacter baumannii is a significant opportunistic nosocomial pathogen responsible for variety of infections, including pneumonia, bloodstream infections, endocarditis, meningitis and infections of the urinary tract, skin and soft tissue $(1,2)$. Reports of the numerous outbreaks caused by multidrug-resistant (MDR) A. baumannii from different regions of the world are appearing at a startling rate, posing an increased threat to hospitalized patients (3-6). Multi-resistance to antibiotics in A. baumannii is greatly exhibited by its intrinsic mechanisms. However, this species shows a remarkable propensity to rapidly acquire and accumulate resistance determinants (7). The resistance mechanisms in A. baumannii are due to the association of diverse mechanisms, including enzymatic modification of antibiotics, low membrane permeability, target gene mutation, and expression of active efflux pumps (8).

Efflux pump systems in bacteria, which can extrude the drugs and toxic compounds by transporting them across the inner and outer membranes into the external environment, have an important role in the development of multidrug resistance (1). ATP-binding cassette (ABC) transporters use ATP as a source of energy, whereas the secondary multidrug transporters are often referred to as H+-drug antiporters, which are sensitive to agents that dispel the proton motive force. To date, nine efflux systems belonging to secondary multidrug transporters have been described in A. baumannii: AdeABC, AdeIJK and AdeFGH, belonging to the resistance-nodulation cell division (RND) and conferring resistance to a wide spectrum of antimicrobial agents $(1,8,9)$; AbeM, included within the multidrug and toxic compound extrusion (MATE), providing moderate resistance to several compounds (10); Tet (A), Tet (B), CraA and AmvA from the major facili-

Implication for health policy/practice/research/medical education

This study indicates that antimicrobial resistance among Acinetobacter baumannii in Iran has emerged as an important challenge for the Iranian medical community. The ability of this organism to rapidly acquire resistance to antimicrobial agents is a cause for great concern. One of the important factors in the multiple resistance phenotype of this problematic organism is active extrusion by efflux pumps. Therefore, there is a need for detailed understanding of the multi-resistance phenotypes of this organism, with attempts to combat these mechanisms by developing inhibitors.

Copyright @ 2013, Ahvaz Jundishapur University of Medical Sciences; Licensee Kowsar Ltd. This is an Open Access article distributed under the terms of the Creative Commons Attribution License (http://creativecommons.org/licenses/by/3.0), which permits unrestricted use, distribution, and reproduction in any medium, provided the original work is properly cited. 
tator superfamily (MFS), involved in tetracycline, minocycline, chloramphenicol and erythromycin resistance, respectively $(7,11,12)$; and AbeS, which is included within the small multidrug resistance (SMR) family (6).

A. baumannii was identified among the top seven problematic hospital-associated pathogens by the Infectious Diseases Society of America (IDSA) and as a critical example of unmet medical need (13). Much more is recognized about efflux systems in Escherichia coli or Pseudomonas aeruginosa than about those in Acinetobacter spp. In spite of the increased frequency of MDR A. baumannii isolates in Iran, there exists a few data regarding the antimicrobial resistance in these Gram negative bacteria. Furthermore, the role of efflux pump mechanism was never examined in Iran.

\section{Objectives}

This study aimed to investigate the role of proton motive force (PMF)-dependent efflux in the primary resistance among clinical isolates of A. baumannii, using carbonyl cyanide m-chlorophenylhydrazone (CCCP) as an efflux pump inhibitor.

\section{Materials and Methods}

\subsection{Bacterial Isolates}

A total of 65 consecutive, non-duplicated A. baumannii clinical isolates were collected from different clinical sources including urine, blood and burn wounds from hospitals in Tehran, Iran. The majority of strains were isolated from burn wounds of hospitalized patients. Definitive identification of A. baumannii was performed by standard biochemical reactions following the criteria of Bouvet and Grimont (14). P. aeruginosa ATCC 27853, Staphylococcus aureus ATCC 25923 and E. coli ATCC 25922 were used as quality control strains for antibiotic susceptibility testing. A. baumannii AB50 was a non-MDR strain from our collection, which displayed susceptibility to fluoroquinolones, $\beta$-lactams and aminoglycosides and was used as a reference strain for comparative analyses.

\subsection{Antimicrobial Susceptibility Testing}

A. baumannii strains were examined for antibiotic resistance by disc diffusion on Mueller-Hinton agar as recommended by the Clinical and Laboratory Standards Institute (15). In brief, an inoculum equivalent to 0.5 McFarland was used to inoculate Mueller-Hinton agar plates, and subsequently discs containing the following antibiotics were added: amikacin $(30 \mu \mathrm{g})$, ampicillinsulbactam $(10 / 10 \mu \mathrm{g})$, ceftazidime $(30 \mu \mathrm{g})$, cefepime (30 $\mu \mathrm{g})$, ciprofloxacin $(5 \mu \mathrm{g})$, ofloxacin $(5 \mu \mathrm{g})$, colistin $(10 \mu \mathrm{g})$, cotrimoxazole $(1.25 / 23.75 \mu \mathrm{g})$, gentamicin $(10 \mu \mathrm{g})$, imipenem $(10 \mu \mathrm{g})$, kanamycin $(30 \mu \mathrm{g})$, piperacillin $(100 \mu \mathrm{g})$ and tobramycin $(10 \mu \mathrm{g})$. Plates were incubated at $37^{\circ} \mathrm{C}$ for $24 \mathrm{~h}$. Multidrug resistance was defined as resistance to three or more representatives of the following classes of antibiotics (16): quinolones (ciprofloxacin and ofloxacin), extended-spectrum cephalosporins (ceftazidime and cefepime), aminoglycosides (amikacin, tobramycin, gentamicin, kanamycin), sulfonamides (cotrimoxazole), penicillins (ampicillin-sulbactam, piperacillin), polymyxin E (colistin) and carbapenems (imipenem). The minimum inhibitory concentrations (MICs) of ciprofloxacin (Temad, Iran), ofloxacin (Zhejiang Youngning, China), ceftazidime (Hanmi, Korea), cefepime (Orchid, India), amikacin (Dobfar, Italy), and gentamicin (Jiangxi, China) were determined by broth microdilution method in triplicate in cation-adjusted Mueller-Hinton broth following the Clinical and Laboratory Standards Institute method (17).

To assess the presumed role of the drug efflux mechanism involved in multidrug-resistance of clinical isolates of A. baumannii, ciprofloxacin MICs were repeated in the presence and absence of the protonophore, carbonyl cyanide m-chlorophenylhydrazone (ССCP; Sigma-Aldrich, Dorset, UK) at a final concentration of $30 \mu \mathrm{M}$. To show that this concentration of CCCP had no intrinsic antibacterial activity against $A$. baumannii, the interference of CCCP was examined by the growth of A. baumannii clinical strains in antibiotic free broth.

\subsection{Accumulation of Ethidium Bromide}

The accumulation of ethidium bromide (EtBr) was determined for MDR clinical strains and a pan-susceptible strain (AB50) as a wild type strain, following the previously described method (2). Briefly, cells were grown overnight, harvested, and resuspended to an $\mathrm{A600}$ of 0.2 in sodium phosphate buffer ( $\mathrm{pH} 7.0$ ) and returned to $37^{\circ} \mathrm{C}$. EtBr was added at a final concentration of $2 \mu \mathrm{g} / \mathrm{ml}$, and after $420 \mathrm{~s}$ of incubation, CCCP was added at a final concentration of $100 \mu \mathrm{M}$ and incubated for a further $180 \mathrm{~s}$. Fluorescence of the samples was measured at excitation and emission wave length of $530 \mathrm{~nm}$ and $600 \mathrm{~nm}$ respectively, using a Shimadzu RF 5000 spectrofluorometer (Shimadzu scientific instruments, Inc, Columbia, Md.). Cells equilibrated with sodium phosphate buffer without EtBr were used as blanks. The results were expressed as percent increased in level of EtBr accumulation after CCCP exposure.

\subsection{Fluoroquinolone Accumulation Studies}

The accumulation of ciprofloxacin was measured for MDRclinical strains showing an MIC of $\geq 4 \mu \mathrm{g} / \mathrm{mL}$ against ciprofloxacin as described previously (18). Ciprofloxacin was added to a final concentration of $10 \mu \mathrm{g} / \mathrm{mL}$, and the fluorescence intensity of the supernatant was measured using a spectrofluorimeter (Shimadzu scientific instruments, Inc, Columbia, Md.) at excitation and emission wavelengths of 279 and $447 \mathrm{~nm}$, respectively. Carbonyl cyanide m- chlorophenylhydrazone (CCCP) was added to a final concentration of $100 \mu \mathrm{M}$ in this experiment. 
Nikasa P et al.

\section{Results}

\subsection{Antimicrobial Resistance Patterns of the Iso- lates}

Biochemical and regular methods enabled the identification of all isolates as A. baumannii . The resistance rates of the 65 strains of $A$. baumannii against piperacillin, kanamycin, cotrimoxazole, tobramycin, ampicillin-sulbactam, colistin and imipenem were 96.9\%, 93.8\%, 95.4\%, $73.8 \%, 47.6 \%, 1.5 \%$ and $44.6 \%$, respectively. The MDR A. bau- mannii detected by agar disc diffusion testing, displayed high levels of resistance to tested antibiotics by broth microdilution method. The antimicrobial susceptibility profiles of the 65 strains for six antibiotics are shown in Table 1 . When CCCP $(30 \mu \mathrm{M})$ was added in ciprofloxacin, the MIC50 decreased four-fold. The results of MICs in the presence and absence of CCCP for four representative MDR strains, namely, $\mathrm{AB} 07, \mathrm{AB} 62, \mathrm{AB} 10, \mathrm{AB} 28$, and one sensitive strain AB50 are shown in Table 2. The reduction in the level of MICs in the presence of CCCP suggests the involvement of proton gradient dependent efflux system(s) in these MDR strains.

Table 1. Antimicrobial Susceptibility Profiles of 65 A.baumannii

\begin{tabular}{lllllll}
\hline Antimicrobial & CIP $^{\mathrm{a}}$ & OFX $^{\mathrm{a}}$ & GEN $^{\mathrm{a}}$ & AMK $^{\mathrm{a}}$ & CAZ $^{\mathrm{a}}$ & FEP $^{\mathrm{a}}$ \\
\hline MIC range $(\mu \mathrm{g} / \mathrm{mL})$ & $\leq 1-512$ & $\leq 2-128$ & $\leq 4-2048$ & $\leq 16-1024$ & $\leq 8-2048$ & $\leq 8-1024$ \\
$\mathbf{M I C 5 0}^{\mathrm{a}}(\mu \mathrm{g} / \mathrm{mL})$ & 32 & 8 & 1024 & 256 & 1024 & 128 \\
$\mathbf{M I C 9 0}^{\mathrm{a}}(\mu \mathrm{g} / \mathrm{mL})$ & 64 & 16 & 2048 & 512 & 2048 & 512 \\
Susceptible, $\%$ & 7.7 & 9.2 & 10.8 & 7.7 & 6.2 & 7.7 \\
Intermediate, \% & 12.3 & 12.3 & 3.1 & 9.2 & 1.5 & 1.5 \\
Resistant, \% & 80 & 78.5 & 86.1 & 83.1 & 92.3 & 90.8
\end{tabular}

a Abbreviations: AMK, amikacin; CAZ, ceftazidime; CIP, ciprofloxacin; FEP, cefepime; GEN, gentamicin OFX, ofloxacin; MIC, minimum inhibitory concentration; MIC

Table 2. Ciprofloxacin MIC Results in the Presence and Absence of CCCP $(30 \mu \mathrm{M})$ Against Four Representative MDR A.baumannii, Namely, AB07, AB62, AB10 and AB28 and a Susceptible Laboratory Strain (AB50)

\begin{tabular}{|c|c|c|c|c|c|}
\hline \multicolumn{6}{|c|}{ MIC $^{\mathrm{a}}(\mu \mathrm{g} / \mathrm{mL})$} \\
\hline Drug & AB10 & AB28 & ABO7 & AB62 & AB50 \\
\hline CIP ${ }^{\mathrm{a}}$ & 32 & 64 & 32 & 64 & 0.25 \\
\hline $\mathrm{CIP}+\mathrm{CCCP}^{\mathrm{a}}$ & 8 & 16 & 16 & 16 & 0.25 \\
\hline
\end{tabular}

a Abbreviations: CCCP, carbonyl cyanide m-chlorophenylhydrazone; CIP, ciprofloxacin; MIC, minimum inhibitory concentration

\subsection{In vitro Studies to Illuminate the Occurrence of Active Efflux}

Multidrug-resistant strains were tested for their ability to EtBr and ciprofloxacin accumulation. The antibioticsusceptible control strain (AB50) showed low increase in EtBr accumulation after the addition of CCCP (38\%). However, the level of accumulation of EtBr in MDR clinical strains increased significantly and ranged from $151 \%$ to 248\% (Figure 1). The representative MDR strains, namely AB10, AB28, AB07 and AB62, showed 173\%, 248\%, 151\% and $164 \%$ increase in EtBr accumulation after the addition of CCCP, respectively (data for other MDR strains are not shown). Ranges of results obtained for the three independent experiments are shown by error bars.
Figure 1. Percent Increases in Ethidium Bromide (EtBr) Accumulation After Exposure to Carbonyl Cyanide m-Chlorophenylhydrazone $(100 \mu \mathrm{M})$ in Four Representative MDR A. baumannii (AB07, AB62, AB10 and AB28) and a Susceptible Laboratory Strain (AB50).

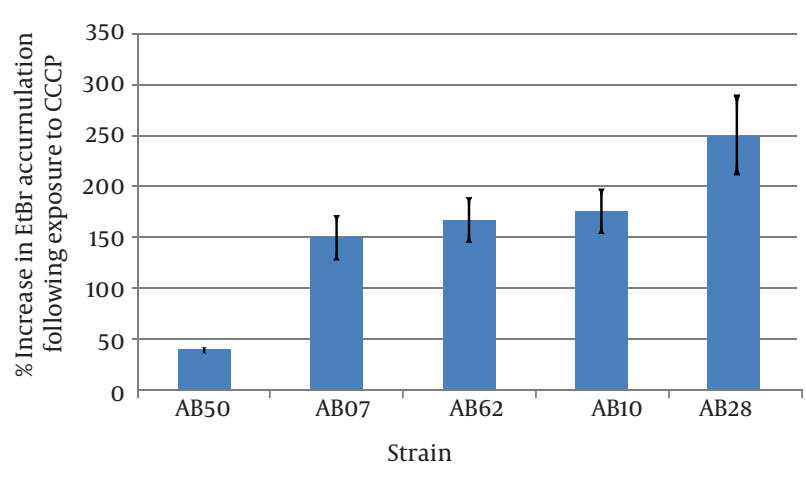

Error bars represent standard errors 
Nikasa P et al.

To elucidate the role of active efflux ciprofloxacin accumulation studies were performed, and analysis revealed that accumulation of ciprofloxacin in sensitive strain (AB50) at steady state was 2.0-3.0 times higher than the amount in MDR strains. The results (Figure 2) showed an increase (2.5-3.0 fold) in the fluorescence intensity in the presence of CCCP $(100 \mu \mathrm{M})$.

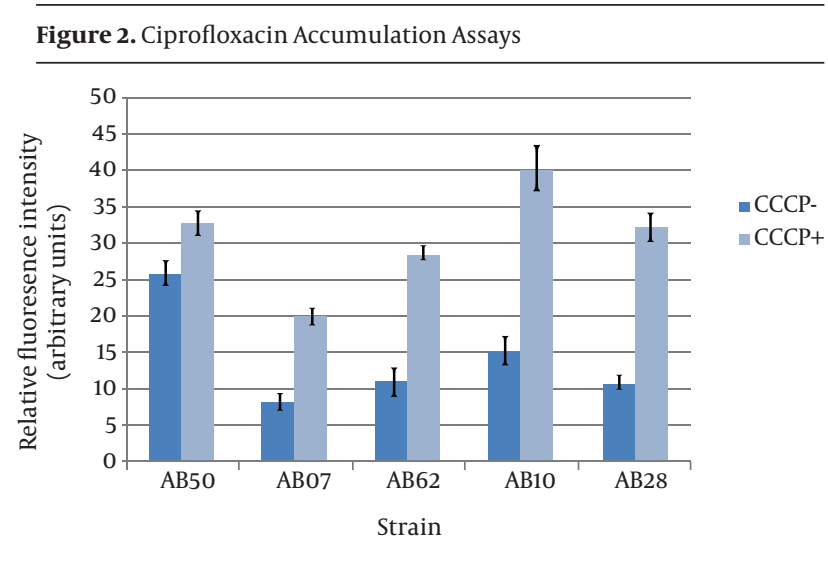

The fluorescence intensity of the supernatant was measured with spectroflourimeter in the presence and absence of efflux pump inhibitor carbonyl cyanide m-chlorophenylhydrazone (CCCP). The results for four representative MDR A. baumannii (AB07, AB62, $\mathrm{AB} 10$ and $\mathrm{AB} 28$ ) and one sensitive strain $A B 50$. All experiments were carried out at least three times

\section{Discussion}

Hospital-acquired infections caused by multidrug-resistant A. baumannii strains have increased considerably to become a significant health problem (19). Recently, the spread of antimicrobial resistance among A. baumannii in Iran has emerged as an important challenge to the medical community. Unfortunately, few data exist regarding to antimicrobial susceptibility of Iranian isolates of $A$. baumannii or on the mechanisms of drug resistance in Iran and most of the Middle Eastern countries. Present findings showed that the resistance in the population of A. baumannii to different antimicrobial drugs in Iran is higher than that of other countries (20-22).

Colistin with susceptibility rate of $98.5 \%$ proved to be the most active antibiotic against these strains, similar to those recently reported (20). In comparison, imipenem, a broad spectrum antibiotic with a better toxicity profile, showed a $55.4 \%$ susceptibility rate. Lower resistance rates to imipenem were reported from Saudi Arabia, Japan and Turkey (about 3-10\%) (23-25). Carbapenem-resistance among clinical $A$. baumannii strains is alarmingly increasing (26), and several studies have shown that MDR A. baumannii are resistant to the most available antibiotics except colisitin. However, colistin is associated with doserelated nephrotoxicity and neurotoxicity (27-29).

To decipher whether the high resistance to tested an- tibacterial agents are due to active efflux mechanism, screening for a potential efflux phenotype was carried out by the determination of the MICs of ciprofloxacin for strains showing an MIC of $\geq 4 \mu \mathrm{g} / \mathrm{mL}$ in the presence and absence of efflux pump inhibitor, CCCP (an uncoupler of oxidative phosphorylation which disrupts the proton gradient on the membrane). CCCP decreased the MICs 2-4 fold, which is consistent with previous report (6). Reduction in the level of MICs in the presence of CCCP demonstrated the presence of proton gradient dependent efflux pump (s) in our isolates. To further assess the role of an efflux-based mechanism in the export of toxic compounds, we performed a functional experiment of efflux pump activity using EtBr and ciprofloxacin accumulation assay in the absence and presence of the efflux pump inhibitor CCCP.

EtBr, as an efficient and common substrate for multidrug transporters, shows high fluorescence intensity under UV light when bound to DNA. While being extruded from the cell through an efflux pump, the fluorescence declines, and this variation can be determined with a spectrofluorometer. In the presence of CCCP, accumulation of EtBr increased 2.5-3.5 fold. Indeed, CCCP disrupts the proton gradient and therefore inhibits efflux pump activity, resulting in an increased EtBr accumulation. This result is consistent with previous reports $(1,2)$. Accumulation assays of ciprofloxacin in the presence of CCCP increased 2.5 to 3.0 fold, suggesting that efflux pump(s), responsible for the reduction of the fluoroquinolone, energized by PMF were functional for drug resistance. A similar response has been reported previously (30). Although efflux pumps play an important role in antibiotic resistance, other resistance mechanisms such as membrane impermeability or enzymatic inactivation may be involved to cause high-level resistance to fluoroquinolones in these MDR A. baumannii strains.

In conclusion, MDR strains of $A$. baumannii have emerged as an important nosocomial pathogen in hospitalized patients. The ability of A. baumannii to rapidly acquire resistance to many commonly prescribed antibiotics is a cause for great concern to clinicians. Practically, now we are closer to the end of the antibiotic era with MDR A. baumannii strains than with methicillin-resistant Staphylococcus aureus $(31,32)$; therefore, there is a clinical need for antibiotic adjuvants with an efflux pump inhibitor activity. The characterization of resistance mechanisms among A. baumannii strains favours the potential development of inhibitors to be used in combination with antimicrobial agents in order to combat this problematic organism. The findings of the present study provide data in favour of active efflux as a significant mechanism in the multiple resistance phenotype of clinical isolates of A. baumannii for the first time in Iran.

\section{Acknowledgements}

None declared. 
Nikasa P et al.

\section{Authors' Contribution}

None declared.

\section{Financial Disclosure}

None declared.

\section{Funding/Support}

This work was supported by a research grant from AlZahra University, Tehran, Iran

\section{References}

1. Magnet S, Courvalin P, Lambert T. Resistance-nodulation-cell division-type efflux pump involved in aminoglycoside resistance in Acinetobacter baumannii strain BM4454. Antimicrob Agents Chemother. 2001;45(12):3375-80.

2. Peleg AY, Adams J, Paterson DL. Tigecycline Efflux as a Mechanism for Nonsusceptibility in Acinetobacter baumannii. Antimicrob Agents Chemother. 2007;51(6):2065-9.

3. Adams-Haduch JM, Paterson DL, Sidjabat HE, Pasculle AW, Potoski BA, Muto CA, et al. Genetic basis of multidrug resistance in Acinetobacter baumannii clinical isolates at a tertiary medical center in Pennsylvania. Antimicrob Agents Chemother. 2008;52(11):3837-43.

4. Kunz AN, Brook I. Emerging resistant Gram-negative aerobic bacilli in hospital-acquired infections. Chemotherapy. 2010;56(6):492-500.

5. Shi WF, Jiang JP, Xu N, Huang ZM, Wang YY. Inhibitory effects of reserpine and carbonyl cyanide m-chloro-phenylhydrazone on fluoroquinolone resistance of Acinetobacter baumannii. Chin Med J (Engl). 2005;118(4):340-3.

6. Srinivasan VB, Rajamohan G, Gebreyes WA. Role of AbeS, a novel efflux pump of the SMR family of transporters, in resistance to antimicrobial agents in Acinetobacter baumannii. Antimicrob Agents Chemother. 2009;53(12):5312-6.

7. Rajamohan G, Srinivasan VB, Gebreyes WA. Molecular and functional characterization of a novel efflux pump, AmvA, mediating antimicrobial and disinfectant resistance in Acinetobacter baumannii.J Antimicrob Chemother. 2010;65(9):1919-25.

8. Damier-Piolle L, Magnet S, Bremont S, Lambert T, Courvalin P. AdeIJK, a resistance-nodulation-cell division pump effluxing multiple antibiotics in Acinetobacter baumannii. Antimicrob Agents Chemother. 2008;52(2):557-62.

9. Cortez-Cordova J, Kumar A. Activity of the efflux pump inhibitor phenylalanine-arginine beta-naphthylamide against the $\mathrm{Ad}$ eFGH pump of Acinetobacter baumannii. Int J Antimicrob Agents. 2011;37(5):420-4.

10. Su XZ, Chen J, Mizushima T, Kuroda T, Tsuchiya T. AbeM, an H+ coupled Acinetobacter baumannii multidrug efflux pump belonging to the MATE family of transporters. Antimicrob Agents Chemother. 2005;49(10):4362-4

11. Marti S, Fernandez-Cuenca F, Pascual A, Ribera A, Rodriguez-Bano J, Bou G, et al. [Prevalence of the tetA and tetB genes as mechanisms of resistance to tetracycline and minocycline in Acinetobacter baumannii clinical isolates]. Enferm Infecc Microbiol Clin. 2006;24(2):77-80

12. Roca I, Marti S, Espinal P, Martinez P, Gibert I, Vila J. CraA, a major facilitator superfamily efflux pump associated with chloramphenicol resistance in Acinetobacter baumannii. Antimicrob Agents Chemother. 2009;53(9):4013-4

13. Talbot GH, Bradley J, Edwards JE, Jr, Gilbert D, Scheld M, Bartlett JG. Bad bugs need drugs: an update on the development pipeline from the Antimicrobial Availability Task Force of the Infectious Diseases Society of America. Clin Infect Dis. 2006;42(5):657-68.

14. Bouvet PJM, Grimont PAD. Identification and biotyping of clini- cal isolates of Acinetobacter. Annales de l'Institut Pasteur / Microbiologie. 1987;138(5):569-578

15. Standard: Clinical Performance Standards for Antimicrobia Disk Susceptibility Tests. Approved Staand Laboratory Standards Institute; 2006 .

16. Wadl M, Heckenbach K, Noll I, Ziesing S, Pfister W, Beer J, et al Increasing occurrence of multidrug-resistance in Acinetobacter baumannii isolates from four German University Hospitals, 2002-2006. Infection. 2010;38(1):47-51.

17. Approved Standard: Clinical and Laboratory Standards Institute. 2006.

18. Srinivasan VB, Virk RK, Kaundal A, Chakraborty R, Datta B, Ramamurthy $\mathrm{T}$, et al. Mechanism of drug resistance in clonally related clinical isolates of Vibrio fluvialis isolated in Kolkata, India. Antimicrob Agents Chemother. 2006;50(7):2428-32.

19. Post V, White PA, Hall RM. Evolution of AbaR-type genomic resistance islands in multiply antibiotic-resistant Acinetobacter baumannii. J Antimicrob Chemother. 2010;65(6):1162-70.

20. Henwood CJ, Gatward T, Warner M, James D, Stockdale MW, Spence RP, et al. Antibiotic resistance among clinical isolates of Acinetobacter in the UK, and in vitro evaluation of tigecycline (GAR-936). J Antimicrob Chemother. 2002;49(3):479-87.

21. Lin L, Ling BD, Li XZ. Distribution of the multidrug efflux pump genes, adeABC, adeDE and adeIJK, and class 1 integron genes in multiple-antimicrobial-resistant clinical isolates of Acinetobacter baumannii-Acinetobacter calcoaceticus complex. Int $J$ Antimicrob Agents. 2009;33(1):27-32.

22. Shi Wei-Feng, Jiang Jian-Ping, Mi Zu-Huang. Relationship between antimicrobial resistance and aminoglycoside-modifying enzyme gene expressions in Acinetobacter baumannii. Chin Med J. 2005;118(2):141-145.

23. Al-Tawfiq JA, Mohandhas TX. Prevalence of antimicrobial resistance in Acinetobacter calcoaceticus-baumannii complex in a Saudi Arabian hospital. Infect Control Hosp Epidemiol. 2007;28(7):870-2.

24. Ishii Y, Alba J, Kimura S, Yamaguchi K. Evaluation of antimicrobial activity of beta-lactam antibiotics by Etest against clinical isolates from 100 medical centers in Japan (2004). Diagn Microbiol Infect Dis. 2006;55(2):143-8.

25. Karsligil T, Balci I, Zer Y. Antibacterial sensitivity of Acinetobacter strains isolated from nosocomial infections. JInt Med Res. 2004;32(4):436-41.

26. Feizabadi MM, Fathollahzadeh B, Taherikalani M, Rasoolinejad M, Sadeghifard N, Aligholi M, et al. Antimicrobial susceptibility patterns and distribution of blaOXA genes among Acinetobacter spp. Isolated from patients at Tehran hospitals. Jpn J Infect Dis. 2008;61(4):274-8.

27. Chusri S, Villanueva I, Voravuthikunchai SP, Davies J. Enhancing antibiotic activity: a strategy to control Acinetobacter infections. JAntimicrob Chemother. 2009;64(6):1203-11.

28. Kwa AL, Loh C, Low JG, Kurup A, Tam VH. Nebulized colistin in the treatment of pneumonia due to multidrug-resistant Acinetobacter baumannii and Pseudomonas aeruginosa. Clin Infect Dis. 2005;41(5):754-7.

29. Mendes RE, Bell JM, Turnidge JD, Castanheira M, Jones RN. Emergence and widespread dissemination of OXA-23, -24/40 and -58 carbapenemases among Acinetobacter spp. in Asia-Pacific nations: report from the SENTRY Surveillance Program.J Antimicrob Chemother. 2009;63(1):55-9.

30. Srinivasan VB, Rajamohan G, Pancholi P, Stevenson K, Tadesse D, Patchanee P, et al. Genetic relatedness and molecular characterization of multidrug resistant Acinetobacter baumannii isolated in central Ohio, USA. Ann Clin Microbiol Antimicrob. 2009;8:21.

31. Giamarellou H, Antoniadou A, Kanellakopoulou K. Acinetobacter baumannii: a universal threat to public health? Int J Antimicrob Agents. 2008;32(2):106-19

32. Hanlon GW. The emergence of multidrug resistant Acinetobacter species: a major concern in the hospital setting. Lett Appl Microbiol. 2005;41(5):375-8. 